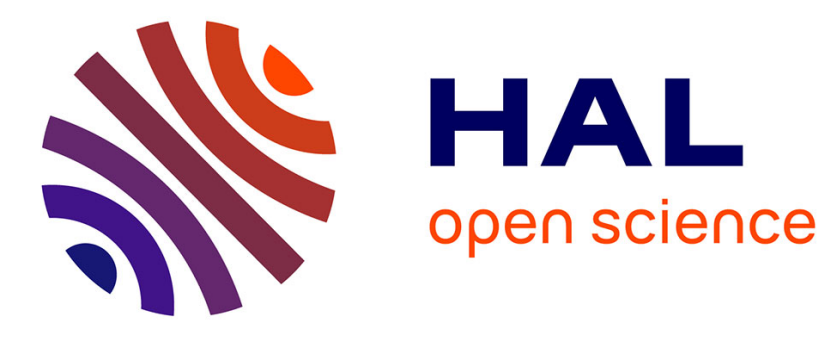

\title{
Packing of sedimenting equiaxed dendrites
}

Antonio Olmedilla, Miha Založnik, Bernard Rouat, Hervé Combeau

\section{To cite this version:}

Antonio Olmedilla, Miha Založnik, Bernard Rouat, Hervé Combeau. Packing of sedimenting equiaxed dendrites. Physical Review E , 2018, 97 (1), 10.1103/PhysRevE.97.012910 . hal-02381423

\section{HAL Id: hal-02381423 \\ https://hal.univ-lorraine.fr/hal-02381423}

Submitted on 8 Jan 2021

HAL is a multi-disciplinary open access archive for the deposit and dissemination of scientific research documents, whether they are published or not. The documents may come from teaching and research institutions in France or abroad, or from public or private research centers.
L'archive ouverte pluridisciplinaire HAL, est destinée au dépôt et à la diffusion de documents scientifiques de niveau recherche, publiés ou non, émanant des établissements d'enseignement et de recherche français ou étrangers, des laboratoires publics ou privés.

$$
\text { Copyright }
$$




\title{
Packing of sedimenting equiaxed dendrites
}

\author{
Antonio Olmedilla, ${ }^{*}$ Miha Založnik, ${ }^{\dagger}$ Bernard Rouat, ${ }^{\ddagger}$ and Hervé Combeau ${ }^{\S}$ \\ Institut Jean Lamour, CNRS, Université de Lorraine, Nancy, France
}

(Received 6 September 2017; published 24 January 2018)

\begin{abstract}
The packing of free-floating crystal grains during solidification has a strong impact on the phase-change process as well as on the structure and the defects in the solidified material. The packing fraction is affected by the particular dendritic morphology of the grains and by their low inertia resulting from the small density difference between solid and liquid. Understanding the grain packing phenomenon during metal alloy solidification is not experimentally possible since packing is coupled to many other phenomena. We therefore investigate the packing of equiaxed dendrites on a model system, consisting of fixed-shape nonconvex model particles sedimenting in conditions hydrodynamically similar to those encountered in solidifying metals. We perform numerical simulations by a discrete-element model and experiments with transparent liquids in a sedimentation column. The combination of experiments and simulations enables us to determine the packing fraction as a function of (i) the grain morphology, expressed by a shape parameter, and (ii) the hydrodynamic conditions, expressed by the particle Stokes number.
\end{abstract}

DOI: 10.1103/PhysRevE.97.012910

\section{INTRODUCTION}

During solidification of metal alloys, a solid nucleates and grows from liquid in the form of dendritic crystal grains that freely float in the liquid melt. Because in alloys of technical interest the solid phase has a higher density than the liquid, the grains settle and eventually pack at the bottom of the solidifying zone. This happens in a wide variety of industrial solidification processes, for example, in large castings or in welding. The way the grains pack has important consequences on the microstructure of the solidified piece (grain size, shape, crystallographic texture, and formation of secondary solid phases) and on several types of defects (porosity and chemical segregation) that can form during solidification.

Unfortunately, little is known about the packing of dendritic crystal grains. Even the most elementary piece of information on the packing, the packing fraction, is poorly characterized. Because of the particular morphology of the dendrites (nonconvex) packing fractions of spheres or other particles of simple convex shape cannot be used. Models of casting processes, where the packing fraction is one of the key parameters, resort to values in a very wide range, from 0.20 up to 0.637 [1-6]. The choice is mostly justified indirectly, by a good fit of the model prediction to experimental characterizations of the piece, for example, in terms of chemical segregation [3]. The disparities in packing fraction are speculated to be due to differences in grain morphology and in hydrodynamic conditions during the settling of the grains; however, clear understanding is lacking.

Particle packing has historically been an attractive problem in physics, essential for the understanding of the properties of granular media as well as of the atomic structure and state

\footnotetext{
*antonio.olmedilla.aero@gmail.com

${ }^{\dagger}$ miha.zaloznik@univ-lorraine.fr

łbernard.rouat@univ-lorraine.fr

§herve.combeau@univ-lorraine.fr
}

transitions in matter [7]. One of the main concerns has been the determination of the minimum and the maximum packings.

For random arrangements of particles [8], an upper and a lower limit of packing are commonly accepted, known as random close packing (RCP) and random loose packing (RLP), respectively. Extensive work has been done on assemblies of spherical particles. In the case of monodisperse hard noncohesive spheres, the random packing fraction has theoretically been shown to be between 0.536 (RLP) and 0.634 (RCP) [9]. Much less knowledge exists on nonspherical particles and most studies tackle only convex particles [10-12].

Dendritic crystal grains have a nonconvex shape and the packing properties of such particles are strikingly different from convex particles. The packing fraction depends strongly on their topology and various shape parameters. Values as low as $\sim 0.1$ have been reported for dendritelike spiky equiaxed particles [13]. Research on nonconvex particles is scarce and is only emerging $[14,15]$.

Experimentally, the transition between RLP and RCP depends on the protocol used to generate the packing. Generally, looser packings are generated with a lower ratio between particle inertia and dissipation [16]. Random loose packing is commonly generated by particle fluidization in a density-matched fluid [17-21] and RCP by mechanical shaking [22,23].

Viscous dissipation is expected to play an important role in packings resulting from settling of dendritic grains during solidification. The inertia of the grains is low due to the small solid-liquid density difference [e.g., $\left(\rho_{\mathrm{s}}-\rho_{\mathrm{l}}\right) / \rho_{\mathrm{s}}=0.06$ in an Al-Zn alloy].

A determination of the packing fraction directly in a solidifying system is extremely difficult because of the progressing solidification of the interstitial liquid. We therefore perform discrete-element method (DEM) numerical simulations and experiments on a transparent model system. The setup in both the experimental and the numerical model consists of particles of fixed shapes similar to dendritic grains. The model particles are packed by sedimentation under conditions 
hydrodynamically similar to those encountered in metal alloys. With a combination of carefully designed experiments and simulations we are able to determine packing fractions in a range of hydrodynamic conditions and for a variety of particle shapes, both representative of solidification systems. We quantify the influence of the grain morphology, expressed by a shape parameter, and of the hydrodynamic conditions, expressed through the Stokes number, on the random packing fraction.

\section{PACKING OF EQUIAXED DENDRITIC GRAINS}

\section{A. Grain morphology and geometrical models}

Metal alloys most often solidify in the form of dendritic crystal grains. The morphology of equiaxed dendrites growing freely in idealized conditions has been characterized in detail $[24,25]$. In realistic process conditions the grain morphology is affected by interactions (soft impingement) between the growing grains and by convection. The resulting grain morphologies are observed to range from clearly dendritic, with two or three levels of fine ramifications, to coarse dendritic (also called globular or cellular), with only coarse primary branches [26,27]. The grain morphology depends on the chemical composition of the alloy and on the solidification conditions (cooling rate, population density of nucleated grains, and convection). In certain casting processes, morphological transitions [3] or mixed dendritic and globular structures [4,28] can be observed within the same cast piece.

In models of solidification at the mushy-zone scale, dendritic grains are often described by a dendrite envelope [2931], which is a smooth surface connecting the tips of the dendrite branches. The concept of the envelope is used to describe the particular shape of the grain without having to account for all the fine-scale features of the branched structure. Envelopes are also used to define dendritic grains in the numerical and experimental models presented in this paper. To account for the wide variety of grain morphologies, two simplified envelope models with variable shape parameters are employed: a dendrite envelope and a globular envelope. Both models are shown in Fig. 1. They both represent grains with six primary branches growing in $\langle 100\rangle$ directions of a cubic structure. Three geometrical parameters are used to describe each grain morphology. The sphericity $\Psi$ is the ratio of the surface area of a sphere with the same volume as the nonspherical particle (equivalent sphere) to the surface area of the particle. The ratio of the equivalent diameter of the envelope to the widest length of the envelope $\chi$ is defined by the diameter of the equivalent sphere $d_{\text {env }}$ and the widest length of the envelope $l_{\text {env }}$ (the length of the line segment that joins the tip of two branches and passes through the envelope center). The third parameter $\eta$ is the ratio of the equivalent diameter of the principal cross section to the perimeter of the principal cross section. The principal cross section is the section made by the plane that contains the envelope center and four branch tips. Note that both envelope models have octahedral symmetry.

(i) The dendrite envelope model [see Fig. 1(a)] is based on six quadrilateral pyramids with an apex angle $\theta$, attached to a

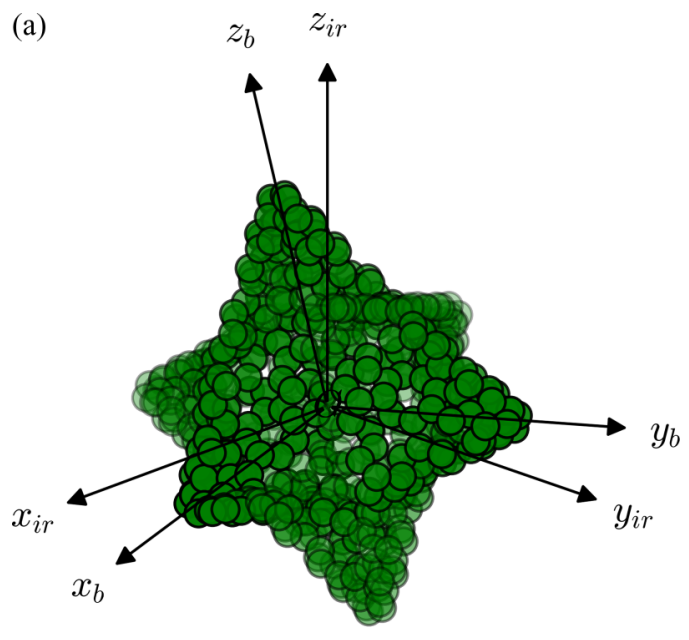

(b)

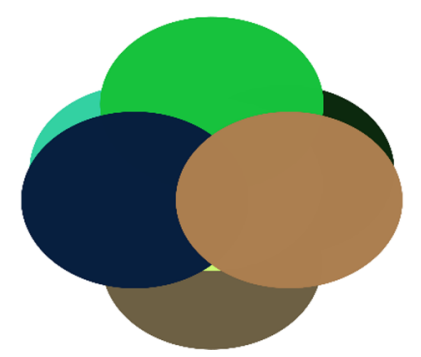

FIG. 1. (a) Model of the dendrite envelope, consisting of six pyramids with an apex angle $\theta$ around a central cube. The surface discretization used in the DEM numerical model is also shown. It consists of 527 clumped spheres arranged on the surface of the particle, internally tangential to the surface. The body and the global coordinate systems are shown to visualize the orientation. (b) Model of the globular envelope, where six clumped spheres form the envelope. The same representation is used in the DEM model.

central cube. The sphericity as a function of $\theta$ is

$$
\Psi(\theta)=\sin \left(\frac{\theta}{2}\right)\left\{\frac{\pi}{6}\left[1+\cot \left(\frac{\theta}{2}\right)\right]^{2}\right\}^{1 / 3} .
$$

The ratio between the equivalent diameter and the widest length $\chi=d_{\mathrm{env}} / l_{\mathrm{env}}$ as a function of $\theta$ is

$$
\chi(\theta)=\frac{\sin \left(\frac{\theta}{2}\right)}{\Psi(\theta)} .
$$

The principal section equivalent diameter to perimeter ratio, which is related to the principal section surface $S_{2 \mathrm{D}}$, hydraulic diameter $D_{\mathrm{H}}$, and perimeter $P$, is

$$
\eta=\left(\frac{4 \pi S_{2 \mathrm{D}}}{P^{2}}\right)^{1 / 2}=\left(\frac{D_{\mathrm{H}}}{P / \pi}\right)^{1 / 2} .
$$

In the particular case of the dendrite envelope model, $\eta$ depends on the apex angle $\theta$ :

$$
\eta(\theta)=\left\{\frac{\pi}{4} \sin \left(\frac{\theta}{2}\right)\left[\sin \left(\frac{\theta}{2}\right)+\cos \left(\frac{\theta}{2}\right)\right]\right\}^{1 / 2} .
$$

Figure 2(a) shows the geometrical properties of the dendrite envelope $\Psi, \eta$, and $\chi$ as a function of the apex angle $\theta$. 
(a)

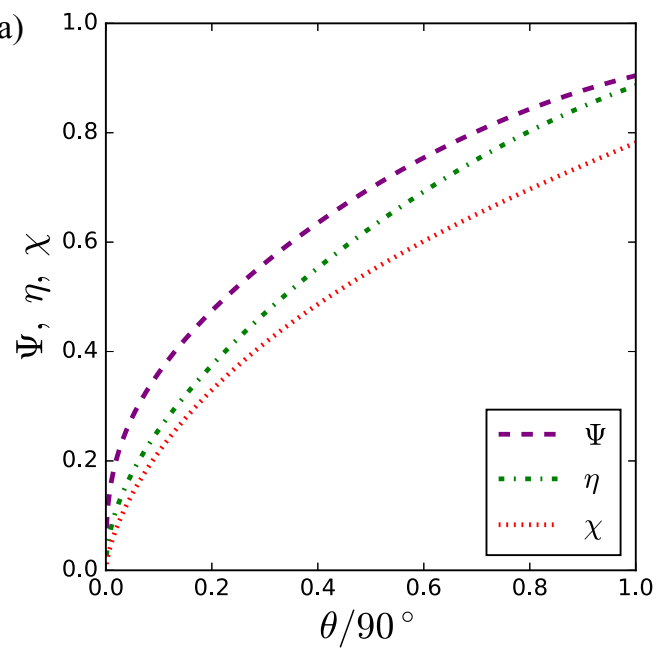

(b)

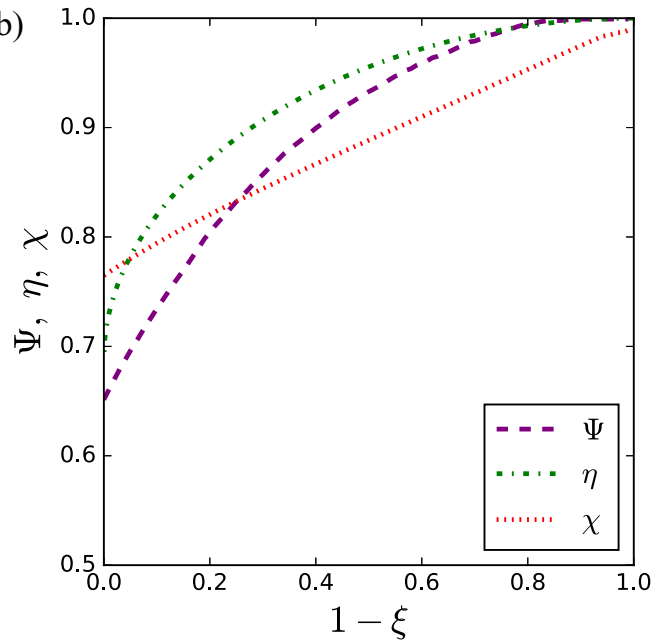

FIG. 2. Geometrical properties $\Psi, \eta$, and $\chi$ for (a) the dendrite envelope, as a function of apex-angle $\theta$, and (b) the globular envelope, as a function of the eccentricity parameter $1-\xi$.

(ii) The globular envelope model is based on six partly overlapping spheres of diameter $d_{\mathrm{mrk}}$ [see Fig. 1(b)]. The center of each sphere $\mathbf{r}_{i}$ with respect to the grain center is given by

$$
\mathbf{r}_{\mathrm{i}}=\frac{\sqrt{2}}{2} \xi d_{\mathrm{mrk}} \mathbf{e}_{\mathrm{i}}, \quad \mathrm{i}=1, \ldots, 6,
$$

where $\mathbf{e}_{i}$ are the unit vectors of the six branch directions and $\xi$ is a dimensionless parameter in the interval $[0,1]$. If $\xi=0$ the six spheres converge to a single sphere, whereas for $\xi=1$ the maximum eccentricity of the spheres is reached and the overlap is reduced to point contacts. The geometrical parameters $\Psi(\xi)$ and $\chi(\xi)$ are computed numerically, whereas $\eta(\xi)$ is expressed analytically:

$$
\eta(\xi)=\left\{\frac{4 \pi\left[\xi^{2}+\xi \cos (\arcsin \xi)+\arcsin \xi+\pi / 4\right]}{(\pi+4 \arcsin \xi)^{2}}\right\}^{1 / 2} .
$$

Figure 2(b) shows the geometrical properties of the globular model $\Psi, \eta$, and $\chi$ as a function of the parameter $1-\xi$.

\section{B. Hydrodynamic conditions}

The dynamics of a particle approaching the packed layer is governed by buoyancy, drag, and lubrication forces. We will present an analysis based on a simple model of a single falling spherical envelope, which shows that the Stokes number is the sole parameter that governs the dynamics of the grain approaching the packed bed. Consequently, the Stokes number should also indicate the dependence of the packing configuration on the inertia of the settling particles. This result agrees with the experiments of Farrell et al. [16], which have shown that the influence of the hydrodynamic conditions of the particle settling on the packing fraction is governed by the Stokes number.

The motion of a single spherical envelope vertically approaching a smooth packed bed, freely falling through a quiescent viscous liquid, is given by

$$
\frac{d}{d t}\left(\rho_{\text {sph }} V_{\text {sph }} \mathbf{v}_{\text {sph }}\right)=\mathbf{F}_{\Delta \mathrm{g}}+\mathbf{F}_{\mathrm{d}}+\mathbf{F}_{\text {lub }}
$$

where $\rho_{\text {sph }}, V_{\text {sph }}$, and $\mathbf{v}_{\text {sph }}$ are the envelope density, volume, and velocity, respectively. On the right-hand side of Eq. (7) we consider the apparent weight $\mathbf{F}_{\Delta \mathrm{g}}=\left(\rho_{\mathrm{sph}}-\rho_{\mathrm{l}}\right) V_{\mathrm{sph}} \mathbf{g}_{0}$, which is the difference between the grain weight and the weight of the liquid of the same volume (with $\rho_{1}$ being the liquid density), the drag force $\mathbf{F}_{\mathrm{d}}$, and the lubrication force $\mathbf{F}_{\text {lub }}$, which is the result of the squeezed fluid layer between the grain and the packed bed.

We assume negligible rotational kinetic energy. We also assume negligible grain growth (i.e., constant grain size) during the approach, the Stokes regime for the drag force $\left(\mathbf{F}_{\mathrm{d}}=-3 \pi d_{\mathrm{sph}} \mu_{1} \mathbf{v}_{\mathrm{sph}}\right)$, and the classical solution of the creeping-flow equations for the lubrication force [32] $\left[\mathbf{F}_{\text {lub }} \approx\right.$ $\left.-(3 / 2) \pi d_{\mathrm{sph}}^{2} \mu_{1} \mathbf{v}_{\mathrm{sph}} / z\right]$, where $\mu_{1}$ is the dynamic viscosity of the liquid and $|z|$ is the gap between the surface of the sphere and the packed bed. We obtain the following equation of motion in dimensionless form:

$$
\frac{d^{2} z^{*}}{d t^{* 2}}+\frac{2}{\mathrm{St}}\left(1+\frac{1}{2 z^{*}}\right) \frac{d z^{*}}{d t^{*}}=-\frac{2}{\mathrm{St}} .
$$

The dimensionless gap is given by $z^{*}=z / d_{\mathrm{sph}}$ and the dimensionless time by $t^{*}=d_{\mathrm{sph}} / v_{\mathrm{sph}}^{\mathrm{sed}}$. The Stokes number St is given by

$$
\mathrm{St}=\left(\frac{\rho_{\mathrm{sph}}}{\rho_{1}}\right)\left(\frac{d_{\mathrm{sph}} v_{\mathrm{sph}}^{\mathrm{sed}}}{9 v_{1}}\right),
$$

where $v_{\mathrm{sph}}^{\mathrm{sed}}$ is the steady-state sedimentation velocity far from the packed bed and $v_{1}$ is the melt kinematic viscosity. A solidifying crystal grain is immersed in liquid of the same material. In metals of industrial relevance the relative density difference between the solid and the liquid phases is generally of only a few percent, i.e., $\rho_{\text {sph }} / \rho_{1} \approx 1$. To estimate the Stokes number in metal alloy solidification, we further estimate the viscosity of liquid metals $v_{1}$ to range from 0.5 up to $2.0 \mathrm{~mm}^{2} / \mathrm{s}$, the grain size $d_{\text {sph }}$ to be between 100 and $1000 \mu \mathrm{m}$, and the sedimentation velocity to range from $o(10)^{-1}$ to $o(100) \mathrm{mm} / \mathrm{s}$. These estimations were obtained from measurements and process simulations of typical casting processes for the production of steel and aluminum alloys $[3,6,33]$. Stokes numbers in the 
interval from $o\left(10^{-3}\right)$ up to $o(10)$ are obtained for these values, which are low inertia-to-dissipation conditions [16].

\section{NUMERICAL MODEL OF PACKING}

\section{A. Description}

A DEM model is used to solve the dynamics of a granular system that simultaneously sediments and packs at the bottom of a square prism domain. The domain walls are semirigid in order to reduce the wall effect. This is done by decreasing the wall stiffness and permitting the particles in contact with the walls to be partially outside the domain, but keeping the center of the particle inside. Our DEM model can account for arbitrary particle geometry and solves for both translation and rotation of each individual particle based on balances of forces and momenta $[34,35]$.

The liquid is not modeled directly. Instead, a special interparticle contact model is used in order to mimic the hydraulic lubrication effects prior to impact between particles. As shown by the estimations in the preceding section, the ratio between the grain inertia and viscous dissipation in solidificationprocess conditions is relatively low [St $\left.\sim o\left(10^{-3}\right) \cdots o(10)\right]$. This means that the lubrication effect dissipates most of the kinetic energy of the grain during its approach, just before impact on the packed bed. Due to this dissipation the effective restitution coefficient upon impact is nil [36]. This is valid for hydrodynamic conditions characterized by a particle Stokes number lower than approximately 10 [36]. In our model the dissipation is reproduced by using critical damping in the classical Kelvin-Voigt inter-particle contact model. Additionally, the grains are ensured to have a low kinetic energy; they are introduced with a very small vertical acceleration $\left(a=10^{-5} g_{0}\right)$. In this way the kinetic energy is dissipated during the contact, which successfully mimics the viscous dissipation due to lubrication in low-St conditions. This contact model does not account for the transformation between liquid and crystal when a pair of crystals enter in contact.

A clumped-sphere model is used to describe the nonspherical morphology of the equiaxed grains. In the case of the dendrite envelopes a surface discretization is carried out by means of small monodisperse nonoverlapping spheres [see Fig. 1(a)]. The spheres are arranged such that the outer tangent planes coincide with the envelope surface. For the globular envelopes, six overlapping monodisperse spheres are used to discretize the volume of the particle. Note that the spheres are only used for contact detection. The mass and the inertia tensor of each particle are defined independently.

Each envelope is characterized by its position and orientation. The positions of the envelopes in the container are initialized by a random sequential addition, whereas the initial random grain orientations are initialized by the Shoemaker algorithm [37].

\section{B. Simulations}

Packings were simulated for six different monodisperse noncohesive frictionless equiaxed dendritic and globular envelope collections, generated with the aforementioned geometric models (Sec. II A). The possible cohesive forces between particles are not considered in this paper, but the role of cohesive
TABLE I. Simulated grain collections.

\begin{tabular}{lc}
\hline \hline Geometry model & Collection characteristics \\
\hline dendrite & 1000 grains, $\theta=45^{\circ}$ (nonconvex) \\
dendrite & 1000 grains, $\theta=60^{\circ}$ (nonconvex) \\
dendrite & 1000 grains, $\theta=90^{\circ}$ (convex, \\
& rhomboid dodecahedron) \\
globular & 1000 grains, $\xi=1.0$ \\
globular & 1000 grains, $\xi=0.8$ \\
globular & 1000 grains, $\xi=0.6$ \\
spherical & 2000 grains, \\
ellipsoidal & 1000 grains, axis ratio $1.25: 0.8: 0.8$ \\
\hline
\end{tabular}

forces in the nonconvex dendrite envelopes is discussed in [35]. Due to the wet contact between the particles (fluid acting as a lubricant), the interparticle friction is neglected in this paper. The effect of the interparticle friction on the packing fraction is studied in detail in [35].

Dendritic particles with three different apex angles and globular particles with three different eccentricities were used. Thus, a wide range of morphologies, in terms of the properties shown in Fig. 2, are represented. Moreover, two other monodisperse noncohesive frictionless collections were packed in order to validate our model by comparison with results from the literature: spheres and revolution ellipsoids (prolate spheroids). The details of the simulated grain collections are presented in Table I. An envelope density of $1400 \mathrm{~kg} / \mathrm{m}^{3}$ and an equivalent diameter of $7 \mathrm{~mm}$ were employed for all collections. The same square-prism simulation domain of $84 \times 84 \times 500 \mathrm{~mm}^{3}$ was used for all collections, except for the spheres. For the spheres the dimensions were $84 \times 84 \times 1000 \mathrm{~mm}^{3}$.

Two examples of packings of dendrite and globular envelopes are shown in Fig. 3. For both collections the three- (a)

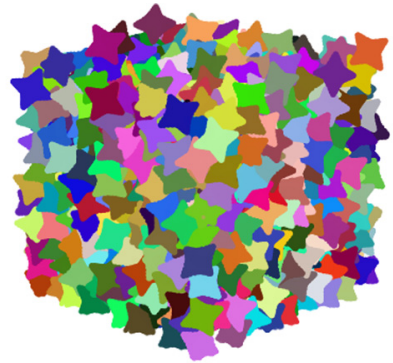

(c)

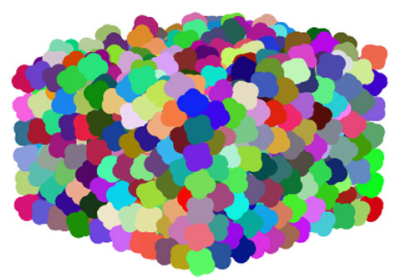

(b)

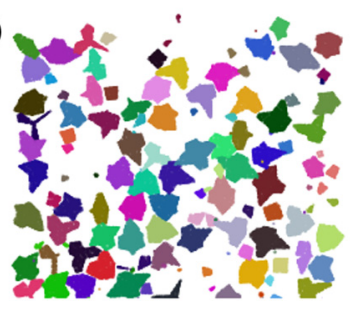

(d)

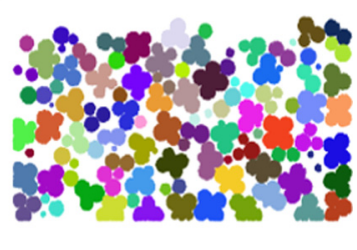

FIG. 3. (a) and (b) Packing of 1000 dendritic envelopes of apex angle $\theta=45^{\circ}$ [a $3 \mathrm{D}$ view and a vertical slice through the packed layer are shown in (a) and (b), respectively]. (c) and (d) Packing of 1000 globular envelopes of $\xi=1.0$ [a $3 \mathrm{D}$ view and a vertical slice of the packed layer are shown in (c) and (d), respectively]. 
dimensional (3D) view of the packing and a 2D view of a vertical slice are shown.

\section{Packing fraction}

Once the granular system achieved a mechanically stable equilibrium, a Voronoi tessellation of the granular system was made by means of the VORO++ open library [38]. The input parameters to build the tessellation are the centers of the envelopes and the walls of the rectangular simulations domain. The sum of the volume of all Voronoi cells corresponds to the volume of the simulation domain: The Voronoi cells of the upper layer of packed particles spread to the top wall of the domain, whereas, for the rest of particles, the Voronoi cells give the particle's local volume.

We compute the volume-averaged packing fraction at a region $\Omega$ as the ratio of the volume of the envelopes whose centers are contained in $\Omega$ to the volume of the Voronoi cells whose corresponding particle centers are contained in $\Omega$ :

$$
\left\langle g_{\text {env }}^{\text {pack }}\right\rangle_{\Omega}=\frac{\sum V_{\text {env }_{\mathrm{i}}}}{\sum V_{\text {cell }_{i}}} \forall \mathrm{i}, \mathbf{r}_{\mathrm{i}} \in \Omega,
$$

where $V_{\mathrm{env}_{\mathrm{i}}}$ and $V_{\text {cell }_{\mathrm{i}}}$ are the volume of the envelope i and the volume of its corresponding Voronoi cell, respectively. The position of the center of the particle $i$ is given by $\mathbf{r}_{\mathrm{i}}$.

We choose the densest inner region of the packing to compute the volume-averaged packing fraction $\Omega$, which is given by the intervals $[22,62] \times[22,62] \times[10,20] \mathrm{mm}^{3}$. In this way, we avoid the wall effect due to the semirigid bottom (within $1.5 d_{\text {env }}$ from the bottom wall) and vertical semirigid walls (a region up to $3 d_{\text {env }}$ ). We also avoid the upper particle layers of the packing that are looser.

In order to quantify the random uncertainty of the simulated packings due to the random initial conditions, the simulations for the spheres and for dendrites of apex angle $\theta=45^{\circ}$ were repeated 10 times. Low relative standard deviations of the volume-averaged packing fraction in $\Omega$ of $0.3 \%$ and $1.5 \%$ were found for the spheres and the dendrites, respectively.

\section{PACKING EXPERIMENTS}

Packing experiments were made for eight different particlefluid combinations in order to investigate the influence of the particle geometry and of the hydrodynamic conditions on the packing fraction. Two monodisperse particle collections (spheres and equiaxed dendrite envelopes of apex angle $\theta=45^{\circ}$ ) were packed by sedimentation in four different fluids: glycerol, a glycerol-water solution, water, and air (dry packing).

\section{A. Setup}

The experimental setup is shown in Fig. 4. It is composed of two 1000-mm-long concentric vertical columns made of transparent acrylic and integrated in a supporting metal structure. The inner column is cylindrical with a $120-\mathrm{mm}$ internal diameter, whereas the outer column has a square cross section with an internal side of $160 \mathrm{~mm}$. Both the cylindrical column and the space between the two columns are filled with the same fluid. This double-wall configuration is designed in order to cancel the horizontal optical deformation of the observed

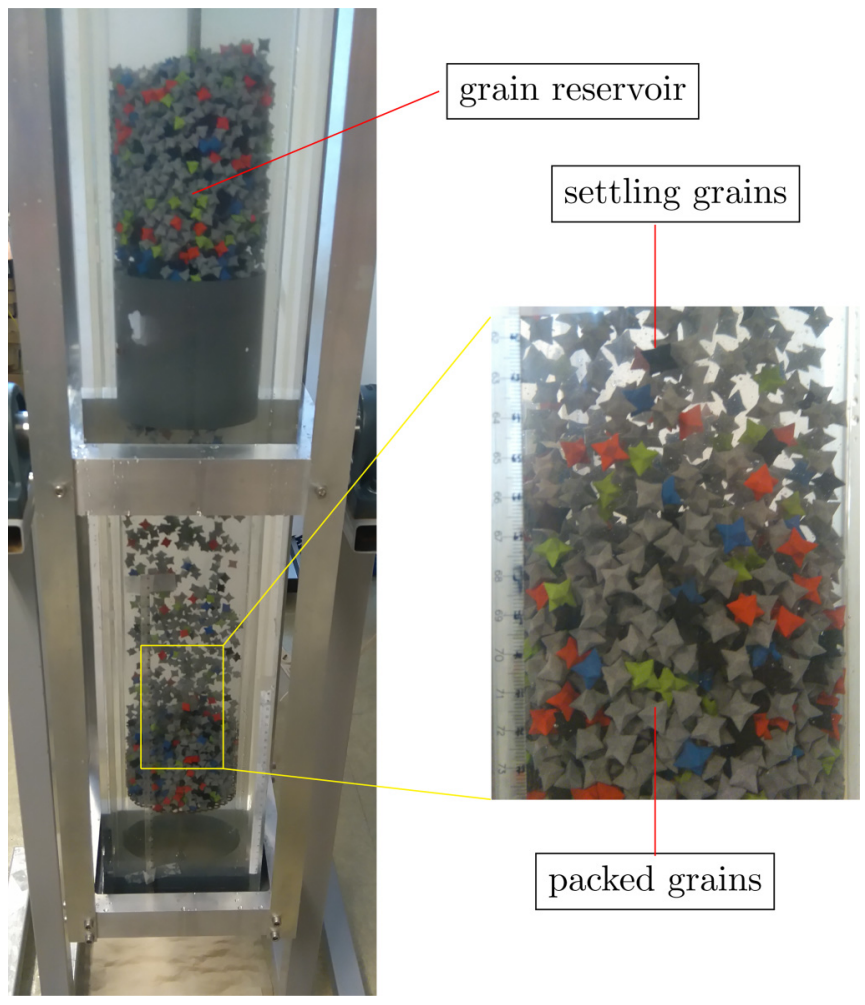

FIG. 4. Shown on the left is the experimental setup with the dendrite collection settling and packing and on the right the zoomed image at the packing zone.

particles due to the refraction on the curved surface of the cylinder. The particles are inserted into the inner cylindrical column. Initially they are contained in the upper part of the column, above the hopper, closed by a gate. The lengths of the upper and the lower parts of the column are 400 and $600 \mathrm{~mm}$, respectively. The whole device volume is filled up with the solid particles and the liquid, avoiding the presence of air. The gate is then opened and the grains pass through the hopper that ensures a constant grain flow. The grains then sediment through the lower part of the column and pack over a horizontal grid.

\section{B. Particles and fluids}

The objective of the particle design and fluid selection is to establish a hydrodynamic similarity between the model experiment and the settling and packing during solidification of metal alloys. The particles (density and size) as well as the fluids (density and viscosity) were chosen in order to have particle Stokes numbers of the same order of magnitude as in metallurgical casting processes (see Sec. IIB).

Two monodisperse noncohesive particle collections were used: spheres and dendrites. The set of spherical particles was composed of approximately 12200 particles, airsoft pellets made of a material based on cornstarch [see Fig. 5(a)]. The sphere size was chosen to have a large container-to-particle diameter ratio $(D / d=20)$ in order to minimize the effect of the container wall on the average packing fraction. This effect is estimated to loosen the average packing fraction of the spheres by less than $2 \%$. This estimation was obtained by integration of the function that describes the variation of the packing fraction 
(a)

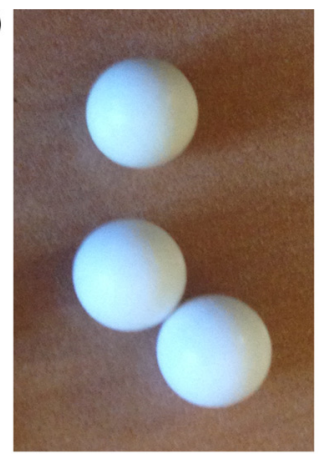

(b)

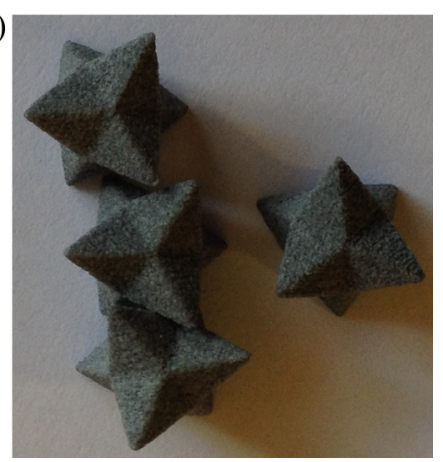

FIG. 5. Grains used in the experiments: (a) spherical particles of $5.92 \pm 0.05 \mathrm{~mm}$ diameter and (b) equiaxed dendrite envelopes of $\theta=45^{\circ}$ of widest length $l_{\text {env }}=12.59 \pm 0.22 \mathrm{~mm}$.

in the vicinity of a container wall as a function of the distance from the wall, determined by Mueller [39]. A wall effect of the same order of the spheres is assumed for the dendrites. This was verified by means of the DEM simulations.

The collection of dendrites was formed by approximately 4400 envelopes [see Fig. 5(b)]. The nonconvex equiaxed particles were made by additive manufacturing. An innovative material based on quasicrystal-polymer composites [40] was used to achieve a target density and to avoid open porosities that could lead to fluid absorption and consequently to particle density variation. The resulting bulk density of the composite material was $\rho_{\mathrm{s}}=1484 \pm 17 \mathrm{~kg} / \mathrm{m}^{3}$, measured by means of a pycnometer according to the protocol in [41]. A sample of 30 nonconvex particles was randomly chosen for mass measurement in a precision balance. The particle mass was $0.3541 \pm 0.0357 \mathrm{~g}$, with a level of confidence of $95 \%$. Their equivalent diameter $d_{\text {env }}$ was $7.70 \pm 0.31 \mathrm{~mm}$ and the widest length $l_{\text {env }}$ was $12.59 \pm 0.22 \mathrm{~mm}$. The density of the spherical particles was similar to that of the dendrites $\left(\rho_{\mathrm{s}}=1391 \pm\right.$ $6 \mathrm{~kg} / \mathrm{m}^{3}$, measured with a pycnometer). The sphere mass was $0.1532 \pm 0.0074 \mathrm{~g}$, with a level of confidence of $95 \%$. The sphere diameter $d_{\text {env }}$ was $5.92 \pm 0.05 \mathrm{~mm}$.

The spherical and dendritic grain collections were packed in four different hydrodynamic conditions provided by four different fluids: pure glycerol, a glycerol-22.3 vol. \% water solution, water, and air. Each fluid provides a different ratio between the particle inertia and the fluid viscous dissipation, expressed by the dimensionless Stokes number $\mathrm{St}=$ $\rho_{\mathrm{s}} d_{\mathrm{env}} v_{\mathrm{s}}^{\text {sed }} / 9 \mu_{\mathrm{f}}$, where $\mu_{\mathrm{f}}$ is the fluid viscosity and $v_{\mathrm{s}}^{\text {sed }}$ is the theoretical settling velocity for a single particle. Table II shows the theoretical Stokes numbers achieved by each protocol (see Appendix A for more details). Despite the glycerol, glycerol-water solution, and water protocols being enough to reproduce the Stokes number range in grain sedimentation

TABLE II. Theoretical Stokes number.

\begin{tabular}{lcccc}
\hline \hline Particle & Glycerol & Glycerol and water & Water & Air \\
\hline sphere & $1.2 \times 10^{-3}$ & 0.5 & 198 & $o\left(10^{5}\right)$ \\
dendrite & $5.0 \times 10^{-3}$ & 1.7 & 368 & $o\left(10^{5}\right)$ \\
\hline \hline
\end{tabular}

during solidification, the air protocol (dry packing) is added in order to widen the range of Stokes numbers.

\section{Packing fraction}

The volume-averaged packing fraction in the packed bed was calculated as the ratio of the total volume of the particles to the total volume occupied by the packed bed $g_{\mathrm{env}}^{\text {pack }}=$ $V_{\mathrm{s}} / V_{\mathrm{t}}$. Similar techniques were often used in the literature $[10,18,19,42]$. The total solid volume $V_{\mathrm{S}}$ was obtained from the total mass and from the solid density measured by the pycnometer $\left(V_{\mathrm{s}}=m_{\mathrm{s}} / \rho_{\mathrm{s}}\right)$. The volume of the packed bed was determined from its height in the cylindrical vessel by reconstruction of the volume from a $2 \mathrm{D}$ image for each packing. Details of the measurement procedure are given in Appendix B.

\section{RESULTS AND DISCUSSION}

\section{A. Influence of the particle shape on the packing fraction: Low Stokes conditions}

The average packing fractions obtained by the DEM simulations (with a protocol that mimics the low Stokes conditions of the experiments in glycerol) are shown as a function of the particle sphericity in Fig. 6. The experimental packings of spheres and dendrites in glycerol are added for comparison with the model. Additionally, our results are compared to data from the literature obtained under similar conditions. Packing fractions of spheres and prolate revolution ellipsoids of axis ratio 1.25:0.8:0.8, obtained by DEM simulations of sedimentation in low inertia-to-dissipation conditions [43], are shown, as well as the packing fractions of nonconvex frictionless particles with six branches (C-shaped particles), packed via a sequential deposition protocol [13].

The influence of the particle shape on the packing is often expressed by the relation of the packing fraction to the particle sphericity, shown in the plot in Fig. 6. From this

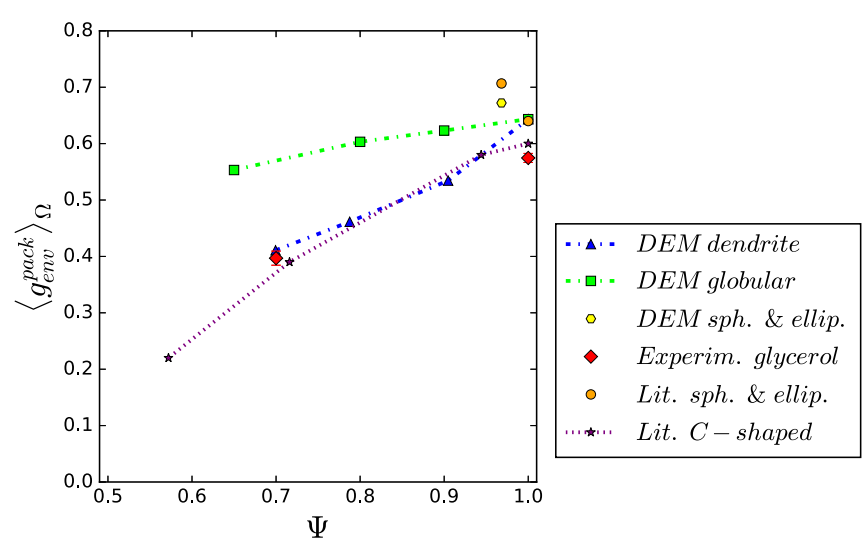

FIG. 6. Averaged packing fraction $\left\langle g_{\text {env }}^{\text {pack }}\right\rangle_{\Omega}$ as a function of the particle sphericity $\Psi$. The experimental sphere and dendrite packings in glycerol are shown with red rhomboid markers. The numerical results from the literature for settling frictionless spheres and revolution ellipsoids of axis ratio 1.25:0.8:0.8 [43] (Lit. sph. \& ellip.) and the numerical results of C-shaped particles packed by sequential deposition under gravity [13] (Lit. C-shaped) are also included. 


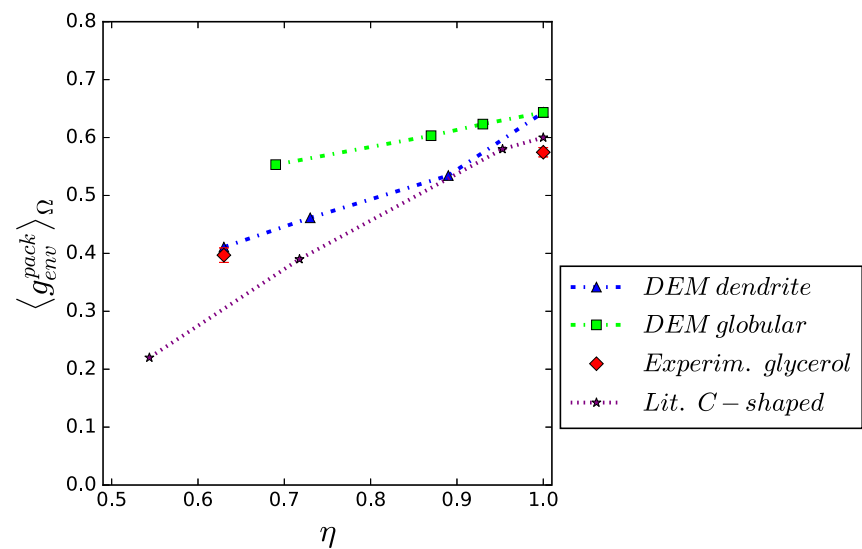

FIG. 7. Average packing fraction $\left\langle g_{\mathrm{env}}^{\text {pack }}\right\rangle_{\Omega}$ as a function of $\eta$. The experimental sphere and dendrite packings in glycerol are shown with red rhomboid markers. The numerical results of hexapod particles (C-shaped) packed by sequential deposition under gravity [13] (Lit. C-shaped) are also included.

classification we can conclude that the packing becomes looser with decreasing envelope sphericity, for both dendritic and globular envelopes. However, different tendencies are found for the dendrite and globular geometries, with a much stronger influence of the sphericity for the dendritic envelopes.

Good agreement between the numerically simulated packing fraction and the experimental packing fractions of the dendrite envelope of $\theta=45^{\circ}(\Psi=0.7)$ is achieved. In the case of the spheres $(\Psi=1)$, a $5 \%$ higher packing fraction is obtained by the DEM model. This difference can be attributed to the importance of the interparticle friction for the sphere packing. Friction is not accounted for in the DEM model. However, our DEM results for spheres and ellipsoids show very good agreement with the DEM simulations of frictionless particles by Delaney et al. [43]. For dendrite envelopes, a similar dependence of the packing fraction on sphericity is found as in the numerical simulations of nonconvex particles (C-shaped particles) by Malinouskaya et al. [13]. Because the packing fractions of dendrite and globular envelopes follow very different trends with sphericity, we can conclude that sphericity is not a universally suitable geometrical property to describe the influence of the nonconvex envelope shape on the packing fraction.

In Fig. 7 the packing fraction of the monodisperse collections is plotted as a function of the parameter $\eta$. In this case, the packing fraction follows different tendencies with $\eta$ for the globular envelopes, dendritic envelopes, and Cshaped particles from [13]. Equivalently to the sphericity, the parameter $\eta$ (geometrical property of the principal cross section of the particle) does not give a more general functional relationship.

Finally, in Fig. 8 the packing fraction is shown as a function of the parameter $\chi$. The packing fraction becomes looser with decreasing $\chi$. Most importantly, the relation of the packing fraction to $\chi$ is very similar for the globular envelopes, dendrite envelopes, and C-shaped particles from [13]. Among the three geometrical parameters studied, $\chi$ seems the best parameter to account for the influence of the shape on the packing fraction for the nonconvex models under study (globular and dendrite

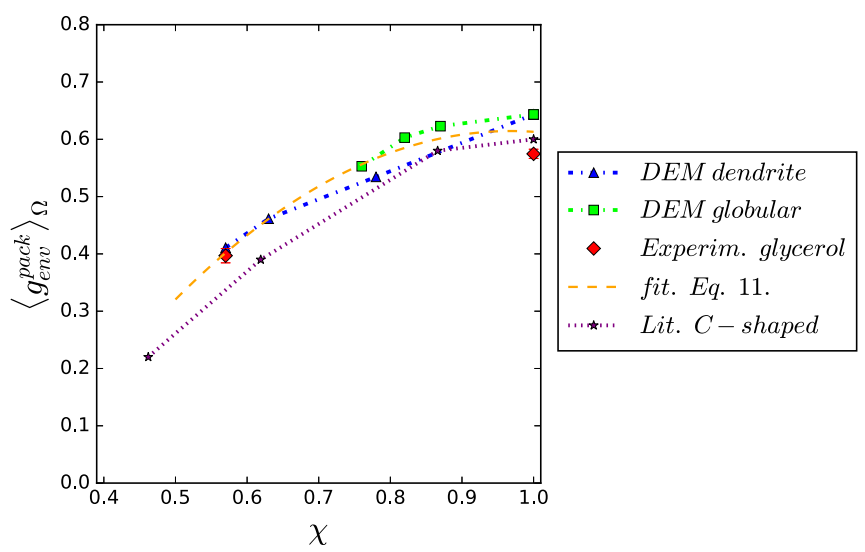

FIG. 8. Average packing fraction $\left\langle g_{\text {env }}^{\text {pack }}\right\rangle_{\Omega}$ as a function of $\chi$. Experimental sphere and dendrite packings in glycerol are shown in red rhomboid markers. The numerical results of hexapod particles (C-shaped) packed by sequential deposition under gravity [13] (Lit. C-shaped) are also included.

envelopes) and $C$ shapes from [13]. This data collapse indicates a single functional relation between $\chi$ and the packing fraction for the three types of hexapod particles.

The shape parameter $\chi$ can relate the nonconvexity of the hexapod particles to their capability to fill up the space in a more general manner than other geometry measures. It seems that the distance between the packed particles is governed by the largest length of the particle branches in a way that is common to all the hexapods investigated in our simulations and in the work by Malinouskaya et al. [13]. For hexapods the parameter $\chi$ appears to reflect the spatial arrangement in the packing in a way somewhat more general than the sphericity, which relates to the particle surface area, or the parameter $\eta$, which relates to the cross section equivalent diameter to perimeter ratio. Based on our data, we propose an empirical relation of $g_{\mathrm{env}}^{\mathrm{pack}}$ as a function of $\chi$, valid for equiaxed hexapod particles:

$$
g_{\text {env }}^{\text {pack }}=0.616+0.084(1-\chi)-1.340(1-\chi)^{2} .
$$

The proposed function tends to the sphere packing as $\chi$ tends to unity in a similar asymptotic manner as indicated by the data from the DEM simulations. In addition, this proposed function follows the same tendency of C-shaped particles of Malinouskaya et al. [13].

\section{B. Influence of the hydrodynamic conditions on the packing fraction}

The influence of the hydrodynamic conditions on the packing was studied by settling experiments in four different fluids. In Fig. 9 the measured packing fractions of spheres (green circles) and dendrites (blue triangles) are shown as a function of the theoretical Stokes number. Each of the experiments is repeated a certain number of times (10 times for glycerol and glycerol-water protocols and 30 times for water and air protocols).

The packings become looser with decreasing Stokes number. A similar tendency of the packing fraction with the Stokes number was found by Farrell et al. [16] for sequential 


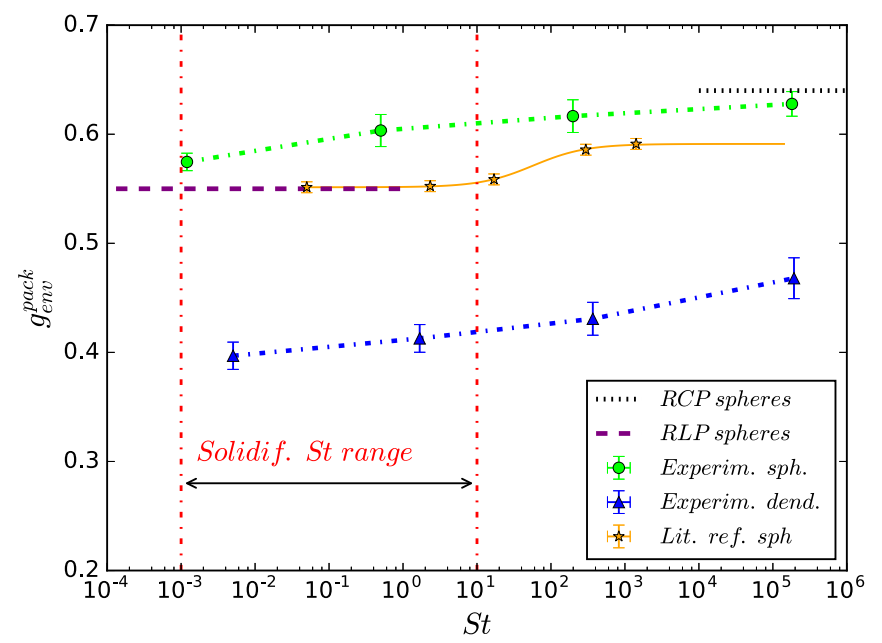

FIG. 9. Average packing fraction $g_{\text {env }}^{\text {pack }}$ as a function of the Stokes number St of our experimental packings of spheres (green circles) and dendrites of apex angle $\theta=45^{\circ}$ (blue triangles). The packing fraction data are generated from repeating each experiment a certain number of times (Sec. B). The experimental RLP [18] (dashed purple line) and RCP (dotted black line) of spheres are also added. The results from [16] (Lit. ref. sph) correspond to a sequential sedimentation of steel monodisperse spheres at different Stokes number. The considerably looser packing fractions are due to the small vessel size as reported by the authors [16]. Solidif. St range refers to the characteristic Stokes number interval in metal alloy solidification.

sedimentation of monodisperse steel spheres (also shown in Fig. 9 for comparison). Note that the packing fractions from Ref. [16] are somewhat lower due to the strong wall effect of the small vessel that was used in that study and possibly due to different interparticle friction coefficients, as reported by the authors.

In the case of spheres, for the studied Stokes range, we find the packing fraction to be between $0.575 \pm 0.008$ and $0.628 \pm$ 0.011 . Our results remain between the RLP $(0.55)$ and the RCP (0.64). However, the packing of spheres that we obtained at the lowest Stokes number $\left(\mathrm{St}=1.2 \times 10^{-3}\right.$ in glycerol) is considerably denser than the loosest experimentally obtained packing of 0.55 [18]. This may be due to a lower Stokes number of sedimentation in [18] since the particles are initially fluidized and after it they sediment and pack in the presence of an upward residual fluid flow that lessens the particle inertia and consequently the Stokes number of sedimentation. On the other hand, the dry packing of spheres provides a result similar to the so-called dry faster pouring of spheres from the literature (0.632 from Ref. [21]).

In the case of the dendrites in the studied Stokes number range, we find the packing fraction to be between $0.397 \pm$ 0.012 and $0.468 \pm 0.018$. The dendrite packings are much looser than sphere packings at all Stokes numbers. The difference is somewhat larger at lower Stokes numbers. The influence of the Stokes number on the packing fraction of dendrites is found to be significant and larger than for spheres. It is also interesting to compare the packing of the nonconvex dendrites to settling-driven packings of convex Platonic solids, which were studied by Baker and Kudrolli [10]. Such a comparison shows that dendrite envelope packings at all St are considerably looser than the loosest packing of any Platonic solid. Notably, the nonconvex dendrites pack about 20\% looser than octahedra, a convex shape often used to approximate dendrite envelopes.

Farrell et al. [16] have shown that the packing fraction of spheres sedimenting in a liquid varies continuously between two limits as a function of the particle Stokes number (for a sequential sedimentation protocol, i.e., the spheres are packed one by one). The low limit is reached when St $\lesssim 10$ and the high limit when $\mathrm{St} \gtrsim 10^{4}$. In our cases of collective sedimentation and packing (of both spheres and dendrite envelopes) a continuous increment of the packing fraction with Stokes number is shown for the hydrodynamic conditions under study. In this way, we show that the collective sedimentation considerably affects the tendency of packing fraction with Stokes number: The transition between supposed lower and upper limits is much wider than in the case of sequential sedimentation. The trend of the packing fraction with the Stokes number is little dependent on geometry for an equivalent collective sedimentation protocol.

\section{CONCLUSION}

We investigated the packing of spherical and nonconvex model particles by sedimentation in fluid. Our model particles have a geometry similar to envelopes of dendritic equiaxed crystal grains that form during solidification of metal alloys and they sediment in conditions hydrodynamically similar to those encountered in metal casting processes. The results can therefore be used for the description of grain packing in such processes. A combination of experiments and DEM numerical modeling was used to investigate the influence of the particular nonconvex shape of the particles and of the hydrodynamic conditions on the packing.

We have shown that the most important influence on the packing is that of the envelope geometry. As the morphology of the particles changes from spherical towards a more nonconvex geometry, the packing fraction decreases. To describe the influence of the nonconvex particle morphology on the packing fraction we proposed the ratio between the equivalent diameter and the widest length of the particle $\chi$.

The influence of the fluid in which the grains settle on the final packing fraction is given by the particle Stokes number. The packing fraction clearly decreases with decreasing Stokes number, due to the lower kinetic energy with which the impacting grains can rearrange the packing. We have shown that grain settling in solidification processes is clearly in the low inertia regime. The packing fractions are thus close to RLP and are only weakly influenced by the variations of St. The influence of hydrodynamic conditions on packing in solidification processes is therefore much smaller than the impact of the particle morphology.

Further work should focus on the influence of particle growth during the settling on packing, improving the contact model in the presence of solid-liquid phase change. More complex hydrodynamic conditions that can be present in solidification processes, such as settling to an inclined packing front and in the presence of a shear flow, should also be investigated. 
TABLE III. Fluid properties.

\begin{tabular}{lcccl}
\hline \hline Property & Glycerol & Glycerol and 22.3 vol \% water & Water & Air \\
\hline$\rho_{\mathrm{f}}\left(\mathrm{kg} / \mathrm{m}^{3}\right)$ & $1262^{\mathrm{a}}$ & $1201^{\mathrm{a}}$ & 1000 & 1.2 \\
$\mu_{\mathrm{f}}(\mathrm{mPa} \mathrm{s})$ & $1180^{\mathrm{a}}$ & 50.4 (measured) & 1.0 & 0.018 \\
\hline \hline
\end{tabular}

${ }^{\mathrm{a}}$ Reference [45].

\section{ACKNOWLEDGMENTS}

This work was supported by the French State through the program "Investment in the future" operated by the National Research Agency and referenced by Program No. ANR-11 LABX-0008-01 (LabEx DAMAS).

\section{APPENDIX A: APPENDIX A: THEORETICAL STOKES NUMBER}

The theoretical Stokes number of the granular system for each fluid protocol is computed by St $=\rho_{\mathrm{s}} d_{\mathrm{env}} v_{\mathrm{s}}^{\text {sed }} / 9 \mu_{\mathrm{f}}$. In the case of glycerol and water protocols, the limit sedimentation velocity is quickly reached. Assuming $g_{\text {env }}^{\text {sed }} \ll g_{\text {env }}^{\text {pack }}$, the influence of multiparticle interactions during settling is neglected, hence $v_{\mathrm{s}}^{\text {sed }}$ is given by the balance of apparent weight and particle drag for a single particle [see Eq. (A1)]

$$
v_{\mathrm{s}}^{\mathrm{sed}}=\left((4 / 3) C_{\mathrm{D}}^{-1}(\operatorname{Re})\left(\frac{\rho_{\mathrm{s}}}{\rho_{\mathrm{f}}}-1\right) g_{\mathrm{o}} d_{\mathrm{env}}\right)^{1 / 2},
$$

where the Reynolds number is given by $\operatorname{Re}=\rho_{\mathrm{f}} d_{\mathrm{env}} v_{\mathrm{s}}^{\mathrm{sed}} / \mu_{\mathrm{f}}$ and the drag coefficient $C_{\mathrm{D}}^{-1}$ is computed assuming spherical geometry with the Oseen correction to the Stokes drag coefficient when $\mathrm{Re}<5$ and the drag coefficient proposed in [44] when $\operatorname{Re} \geqslant 5$.

In the case of settling in air, the grains do not reach the limit velocity within the column height. Drag and buoyancy are negligible and the acceleration is due to gravity only. The settling velocity prior to packing $v_{\mathrm{s}}^{\text {sed }}$ is computed by Eq. (A2) with $h \approx 0.5 \mathrm{~m}$,

$$
v_{\mathrm{s}}^{\mathrm{sed}}=\left(2 g_{\mathrm{o}} h\right)^{1 / 2}
$$

In Table III the fluid properties are collected for each protocol. The viscosity of glycerol depends on temperature and was taken from [45]. The column fluid temperature was measured by means of a thermocouple and was approximately $21{ }^{\circ} \mathrm{C}$ during all experiments. The viscosity of the glycerolwater solution was measured with a falling-ball viscosimeter.

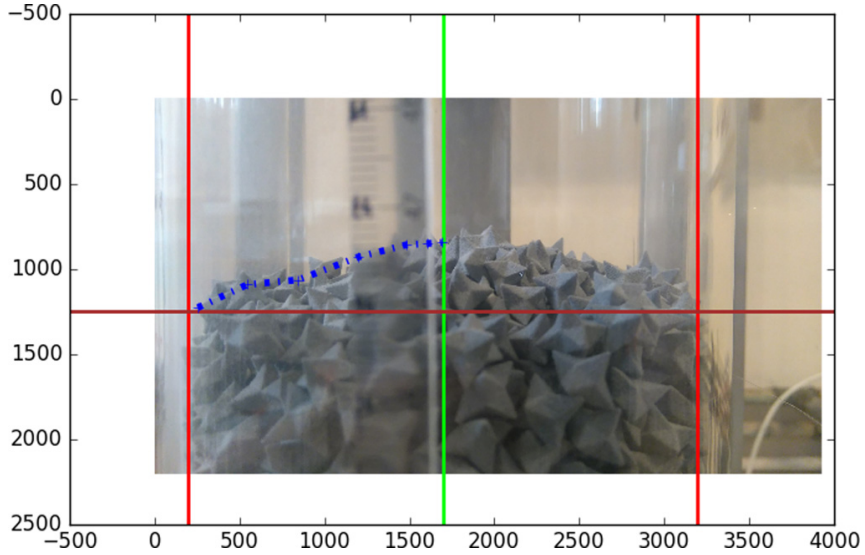

FIG. 10. Estimation of the total occupied volume $V_{\mathrm{t}}$ from a 2D image. Nonconvex dendritic grains are in the image. The horizontal and vertical axes are shown in pixels.

\section{APPENDIX B: APPENDIX B: PACKING FRACTION MEASUREMENT}

The hopper is designed to give the packed granular system a convex top surface. Flatter mound structures are found for glycerol and glycerol-water protocols compared to water and air protocols. The flatter mound may be a consequence of the importance of the lubrication forces between grains for glycerol and glycerol-water solution protocols. In this way the grains easily slip, not being able to form high mound structures.

The observed surface shapes made it reasonable to assume that the surface is axisymmetric and this assumption was used to estimate the total volume occupied by the packing. In order to increase the accuracy of the estimations, a semirevolution model was used. The left and right halves of the surface contour determined from a photograph (see the blue dashed line in Fig. 10) were each used to calculate the volume of an axisymmetric half dome. These volumes were then used to calculate the total volume of the packing. The perspective distortions due to the different distance of the vessel center and sides from the camera were carefully corrected when using the length measurements from the images. The sedimentation and packing experiments were repeated 10, 10, 30, and 30 times for the glycerol, glycerol-water, water, and air protocols, respectively. The uncertainty of the packing fraction measurement includes the measurement uncertainties of the solid volume $V_{\mathrm{s}}$ and total volume $V_{\mathrm{t}}, \frac{\Delta g_{\mathrm{env}}^{\mathrm{pack}}}{\bar{g}_{\mathrm{env}}^{\text {pack }}} \approx \frac{\Delta V_{\mathrm{s}}}{\bar{V}_{\mathrm{s}}}+\frac{\Delta V_{\mathrm{t}}}{\bar{V}_{\mathrm{t}}}$. The solid volume uncertainty is given by the pycnometer uncertainty $\frac{\Delta V_{\mathrm{s}}}{\bar{V}_{\mathrm{s}}} \approx \frac{\Delta \rho_{\mathrm{s}}}{\bar{\rho}_{\mathrm{s}}}$ and $\frac{\Delta V_{\mathrm{t}}}{\bar{V}_{\mathrm{t}}}$ includes both the random character of the packing phenomenon and the measurement error. We assume $\Delta V_{\mathrm{t}}$ to be two standard deviations (level of confidence of $95 \%$ ).
[1] T. Wang, M. Wu, A. Ludwig, M. Abondano, B. Pustal, and A. Bührig-Polaczek, Int. J. Cast Metals Res. 18, 221 (2005).

[2] C. J. Vreeman, J. Schloz, and M. M. Krane, ASME J. Heat Transfer 124, 947 (2002).

[3] H. Combeau, M. Založnik, S. Hans, and P. E. Richy, Metall. Mater. Trans. B 40, 289 (2009).
[4] M. Založnik, A. Kumar, H. Combeau, M. Bedel, P. Jarry, and E. Waz, Adv. Eng. Mater. 13, 570 (2011).

[5] N. Leriche, Étude de la transition colonnaire-equiaxe dans les lingots et en coulée continue dacier et influence du mouvement des grains, Ph.D. thesis, Université de Lorraine, 2015. 
[6] L. Heyvaert, M. Bedel, M. Založnik, and H. Combeau, Metall. Mater. Trans. A 48, 4713 (2017).

[7] L. Berthier, Images Phys., 28 (2011), http://www.cnrs.fr/ publications/imagesdelaphysique/couv-PDF/IdP2011/ 05_Berthier.pdf.

[8] S. Torquato, T. M. Truskett, and P. G. Debenedetti, Phys. Rev. Lett. 84, 2064 (2000).

[9] C. Song, P. Wang, and H. A. Makse, Nature (London) 453, 629 (2008).

[10] J. Baker and A. Kudrolli, Phys. Rev. E 82, 061304 (2010).

[11] A. Jaoshvili, A. Esakia, M. Porrati, and P. M. Chaikin, Phys. Rev. Lett. 104, 185501 (2010).

[12] W. Man, A. Donev, F. H. Stillinger, M. T. Sullivan, W. B. Russel, D. Heeger, S. Inati, S. Torquato, and P. M. Chaikin, Phys. Rev. Lett. 94, 198001 (2005).

[13] I. Malinouskaya, V. V. Mourzenko, J.-F. Thovert, and P. M. Adler, Phys. Rev. E 80, 011304 (2009).

[14] J. Barés, Y. Zhao, M. Renouf, K. Dierichs, and R. Behringer, EPJ Web Conf. 140, 06021 (2017).

[15] C. Avendaño and F. A. Escobedo, Curr. Opin. Colloid Interface Sci. 30, 62 (2017).

[16] G. R. Farrell, K. M. Martini, and N. Menon, Soft Matter 6, 2925 (2010).

[17] G. Y. Onoda and E. G. Liniger, Phys. Rev. Lett. 64, 2727 (1990).

[18] M. Jerkins, M. Schröter, H. L. Swinney, T. J. Senden, M. Saadatfar, and T. Aste, Phys. Rev. Lett. 101, 018301 (2008).

[19] M. Schröter, D. I. Goldman, and H. L. Swinney, Phys. Rev. E 71, 030301 (2005).

[20] T. Aste, T. Di Matteo, M. Saadatfar, T. J. Senden, M. Schröter, and H. L. Swinney, Europhys. Lett. 79, 24003 (2007).

[21] G. W. Delaney, T. Di Matteo, and T. Aste, Soft Matter 6, 2992 (2010).

[22] G. Scott and D. Kilgour, J. Phys. D 2, 863 (1969).

[23] E. R. Nowak, J. B. Knight, E. Ben-Naim, H. M. Jaeger, and S. R. Nagel, Phys. Rev. E 57, 1971 (1998).
[24] J. Gibbs, K. A. Mohan, E. Gulsoy, A. Shahani, X. Xiao, C. Bouman, M. De Graef, and P. Voorhees, Sci. Rep. 5, 11824 (2015).

[25] A. J. Melendez and C. Beckermann, J. Cryst. Growth 340, 175 (2012).

[26] D. Ovsienko, G. Alfintsev, and V. Maslov, J. Cryst. Growth 26, 233 (1974).

[27] Ø. Nielsen, A. Mo, B. Appolaire, and H. Combeau, Metall. Mater. Trans. A 32, 2049 (2001).

[28] D. G. Eskin, R. Nadella, and L. Katgerman, Acta Mater. 56, 1358 (2008).

[29] C. Y. Wang and C. Beckermann, Metall. Mater. Trans. A 27, 2754 (1996).

[30] J. A. Dantzig and M. Rappaz, Solidification, 1st ed. (EPFL, Lausanne, 2009).

[31] Y. Souhar, V. F. De Felice, C. Beckermann, H. Combeau, and M. Založnik, Comput. Mater. Sci. 112, 304 (2016).

[32] H. Brenner, Chem. Eng. Sci. 16, 242 (1961).

[33] H. Combeau, M. Založnik, and M. Bedel, JOM 68, 2198 (2016).

[34] A. Olmedilla, M. Založnik, and H. Combeau, EPJ Web Conf. 140, 06002 (2017).

[35] A. Olmedilla, Grain motion and packing: Application to metallic alloy solidification, Ph.D. thesis, Université de Lorraine, 2017.

[36] E. Izard, T. Bonometti, and L. Lacaze, J. Fluid Mech. 747, 422 (2014).

[37] K. Shoemaker, Comput. Graphics 19, 245 (1985).

[38] C. H. Rycroft, Chaos 19, 041111 (2009).

[39] G. E. Mueller, Powder Technol. 72, 269 (1992).

[40] S. Kenzari, D. Bonina, A. Degiovanni, J.-M. Dubois, and V. Fournée, Acta Phys. Pol. A 126, 449 (2014).

[41] ASTM D854-00, Standard Test Methods for Specific Gravity of Soil Solids by Water Pycnometer, ASTM International, West Conshohocken, PA, 2000, www.astm.org.

[42] I. Higuti, Ann. Inst. Stat. Math. 12, 257 (1961).

[43] G. W. Delaney, J. E. Hilton, and P. W. Cleary, Phys. Rev. E 83, 051305 (2011).

[44] M. Mikhailov and A. S. Freire, Powder Technol. 237, 432 (2013).

[45] N.-S. Cheng, Ind. Eng. Chem. Res. 47, 3285 (2008). 